\title{
Two-dimensional semimetal in a wide HgTe quantum well: Magnetotransport and energy spectrum
}

\author{
G. M. Minkov, ${ }^{1,2}$ A. V. Germanenko, ${ }^{2}$ O. E. Rut, ${ }^{2}$ A. A. Sherstobitov, ${ }^{1,2}$ S. A. Dvoretski, ${ }^{3}$ and N. N. Mikhailov ${ }^{3}$ \\ ${ }^{1}$ Institute of Metal Physics RAS, 620990 Ekaterinburg, Russia \\ ${ }^{2}$ Institute of Natural Sciences, Ural Federal University, 620000 Ekaterinburg, Russia \\ ${ }^{3}$ Institute of Semiconductor Physics RAS, 630090 Novosibirsk, Russia
}

(Received 27 November 2012; revised manuscript received 11 September 2013; published 9 October 2013)

\begin{abstract}
The results of an experimental study of the magnetoresistivity and the Hall and Shubnikov-de Haas effects for a heterostructure with a HgTe quantum well of $20.2 \mathrm{~nm}$ width are reported. The measurements were performed on gated samples over a wide range of electron and hole densities including the vicinity of a charge neutrality point. Analyzing the data, we conclude that the electron and hole energy spectra are in qualitative agreement with those calculated within the framework of $k P$ model. The electron and hole subbands are overlapped due to the nonmonotonic dispersion of the hole subband resulting in a semimetallic state. The main result of the paper, however, is the drastic quantitative difference in the experimental and calculated spectra of the hole subband. So, the hole effective mass found from the analysis of the Shubnikov-de Haas oscillations is positive and equal to approximately $0.2 m_{0}$ and practically independent of the quasimomentum $(k)$ starting from $k^{2} \simeq 0.7 \times 10^{12} \mathrm{~cm}^{-2}$, while the theory predicts negative (electronlike) effective mass up to $k^{2} \simeq 6 \times 10^{12} \mathrm{~cm}^{-2}$. The experimental effective mass near $k=0$, where the hole energy spectrum is electronlike, is close to $-0.005 m_{0}$, whereas the theoretical value is about $-0.1 m_{0}$.
\end{abstract}

DOI: 10.1103/PhysRevB.88.155306

PACS number(s): 73.20.Fz, 73.61.Ey

\section{INTRODUCTION}

Two-dimensional (2D) systems based on gapless semiconductors such as $\mathrm{HgTe}$ represent unique objects. A great variety of two-dimensional electron and hole systems based on these materials can be realized depending on the quantum well width (d) and content of cadmium in the $\mathrm{Hg}_{1-x} \mathrm{Cd}_{x} \mathrm{Te}$ well and $\mathrm{Hg}_{1-y} \mathrm{Cd}_{y} \mathrm{Te}$ barriers. It is now well established that the energy spectrum in $\mathrm{CdTe} / \mathrm{HgTe} / \mathrm{CdTe}$ quantum well at $d=d_{c} \simeq$ $6.5 \mathrm{~nm}$ is gapless ${ }^{1}$ and is close to the linear Dirac-like spectrum at small quasimomentum $k{ }^{2}$ When the thickness $d<d_{c}$ (i.e., when the HgTe quantum well is narrow), the ordering of energy subbands of spatial quantization is analogous to that in conventional narrow-gap semiconductors; the highest valence subband at $k=0$ is formed from the heavy-hole $\Gamma_{8}$ states, while the lowest electron subband is formed both from the $\Gamma_{6}$ states and light-hole $\Gamma_{8}$ states. For a thick HgTe layer, $d>d_{c}$, the quantum well is in the inverted regime; the main electron subband is formed from the heavy-hole $\Gamma_{8}$ states, ${ }^{3}$ whereas the subband formed from the $\Gamma_{6}$ states and light-hole $\Gamma_{8}$ states sinks into the valence band.

The energy spectrum and transport phenomena of 2D carriers in $\mathrm{HgTe}$-based structures were studied intensively last decade both experimentally ${ }^{4-10}$ and theoretically. ${ }^{2,11-13}$ The experimental data on the energy difference between the different $2 \mathrm{D}$ subbands at zero quasimomentum are in satisfactory agreement with the theory. The electron energy spectrum, electron effective mass, and their dependence on the quantum well width are in agreement with the calculation results also. As regards to the experimental data on the valence band energy spectrum, namely, the value of bands overlapping, role of strain, effective masses at $k=0$ and at large quasimomentum, these are mutually contradictory and call for further investigation.

In this paper, we present the results of an experimental study of the transport properties of a heterostructure with a $\mathrm{HgTe}$ quantum well with an inverted energy spectrum. The measurements were performed over a wide range of electron and hole densities including the vicinity of the charge neutrality point (CNP) with nearly equal electron and hole densities. Analysis of experimental data allows us to reconstruct the energy spectrum, which is in qualitative agreement with that calculated theoretically within the framework of standard $k P$ model. The experimental and calculated effective masses of the electron subband are close to each other. The dispersion law $E(k)$ for the valence band is nonmonotonic, which results in the overlap with the conduction band. However, the experimental and calculated spectra being in qualitative agreement are strongly different quantitatively. We show in particular that the maxima in the dispersion are located at $k \lesssim 0.5 \times 10^{6} \mathrm{~cm}^{-1}$, while the theory predicts a value of about $2.5 \times 10^{6} \mathrm{~cm}^{-1}$. Moreover, our estimate for the effective mass of the electronlike part of the valence band spectrum at $k=0$ gives the value of about $0.005 m_{0}$, whereas the calculated value is larger than $0.1 m_{0}$.

The paper is organized as follows. The next section is devoted to the experimental details and sample description. The experimental results and discussion are given in Sec. III. After the short data overview in the beginning, we thoroughly analyze the magnetoresistivity oscillations and the classical magnetoresistivity and Hall effects. In Sec. IV, the reconstruction of the energy spectrum is made, the detailed comparison between the experimental and calculated spectra is carried out. Additional experimental arguments based on the specific features of the Landau quantization in the systems under study, which supports the reconstructed spectrum, are given there as well. Finally, conclusions are presented in Sec. V.

\section{EXPERIMENTAL}

Our $\mathrm{HgTe}$ quantum wells were realized on the basis of $\mathrm{HgTe} / \mathrm{Hg}_{1-x} \mathrm{Cd}_{x} \mathrm{Te}(x=0.58)$ heterostructure grown by molecular beam epitaxy on GaAs substrate with the (013) 

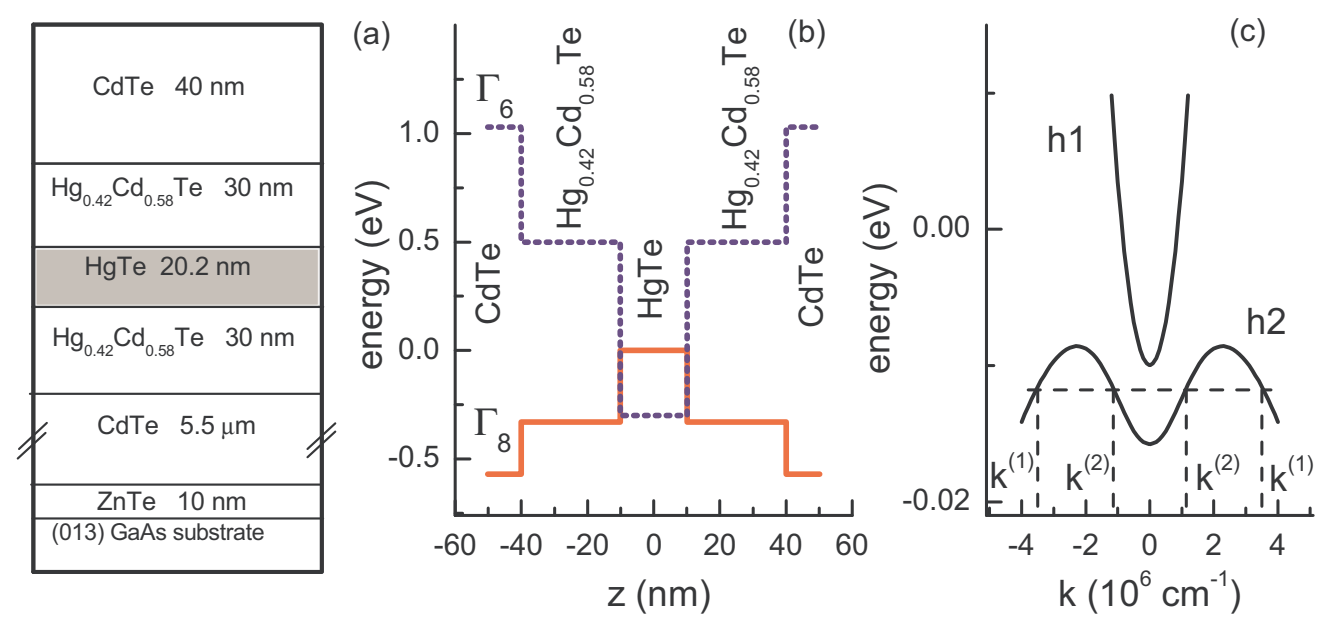

FIG. 1. (Color online) Architecture (a) and energy diagram (b) of the structure under investigation. (c) The energy spectrum calculated within the framework of isotropic six-band $k P$ model.

surface orientation. ${ }^{14}$ The nominal width of the quantum well was $d=20.2 \mathrm{~nm}$. The samples were mesa etched into standard Hall bars of $0.5 \mathrm{~mm}$ or $0.1 \mathrm{~mm}$ width with the distance between the potential probes of $0.5 \mathrm{~mm}$. To change and control the electron and hole densities ( $n$ and $p$, respectively) in the quantum well, the field-effect transistors were fabricated with parylene as an insulator and aluminium as a gate electrode. The measurements were performed at temperature of 1.3-4.2 K in magnetic field up to $8 \mathrm{~T}$. All the data will be presented for $T=1.35 \mathrm{~K}$, unless otherwise specified. The architecture and the energy diagram of the structure investigated are shown in Figs. 1(a) and 1(b), respectively. The energy spectrum calculated within the framework of the six-band $k P$ model with taking into account the lattice mismatch between the $\mathrm{Hg}_{1-x} \mathrm{Cd}_{x} \mathrm{Te}$ layers forming the quantum well and CdTe buffer layer is presented in Fig. 1(c). The calculations have been performed within the framework of isotropic approximation using the direct integration technique as described in Ref. 15. We used the parameters from Refs. 16 and 17. It is seen that although the $\mathrm{h} 1$ and $\mathrm{h} 2$ subbands are separated by a gap of about $5 \mathrm{meV}$ at $k=0$, there is an overlap between them of about $1.5 \mathrm{meV}$ due to the nonmonotonic dispersion $E(k)$ of the h2 subband.

\section{EXPERIMENTAL RESULTS AND DISCUSSION}

\section{A. Data overview}

An overview of the magnetic field dependencies of the transverse $\left(\rho_{x y}\right)$ and longitudinal $\left(\rho_{x x}\right)$ resistivities for different gate voltages $\left(V_{g}\right)$ is presented in Fig. 2. It is seen that the well defined quantum Hall plateaus of $\rho_{x y}$ and minimum of $\rho_{x x}$ are observed at electron $\left(V_{g}>2 \mathrm{~V}\right)$ and hole $\left(V_{g}<-1 \mathrm{~V}\right)$ conductivity regimes. Two important features of these dependencies should be pointed out.

First, some extremum on the $\rho_{x y}$ versus $B$ and $\rho_{x x}$ versus $B$ dependencies at $B=4-6 \mathrm{~T}$ [marked by arrows in Fig. 2(a) and in the inset in Fig. 2(b)] is observed. Its position only slightly depends on $V_{g}$ within the gate voltage range from 0.8 to $3.0 \mathrm{~V}$.

Second, the Hall resistivity $\rho_{x y}$ changes its sign with increasing field at $-3 \mathrm{~V}<V_{g}<1.8 \mathrm{~V}$ [this is better evident in Fig. 3(a)]. Such behavior of $\rho_{x y}$ accompanied by strong positive magnetoresistivity [Fig. 3(b)] is analogous to that observed in the case of two types of carriers that give the electron and holelike contributions.

It is important for further consideration to know the gate voltage dependence of the carrier densities. The first estimates can be already obtained from simplified analysis of the Hall effect data. Notwithstanding the fact that the $B$ dependence of the Hall coefficient $R_{H}=\rho_{x y} / B$ is rather complicated at intermediate gate voltages, there are ranges of the magnetic fields where $R_{H}$ is practically independent of $B$ (see the inset in Fig. 4). For $V_{g}<1 \mathrm{~V}$, this range is $B \simeq 1.5-2.5 \mathrm{~T}$, where $R_{H}$ is positive. For $V_{g}>2 \mathrm{~V}, R_{H}$ being negative is nearly constant at $B \lesssim 0.05 \mathrm{~T}$. So, the densities of electrons and holes could be estimated as $1 / e\left|R_{H}(B)\right|$ at $B=0.05$ and $2 \mathrm{~T}$, respectively. As seen from Fig. $4, n$ and $p$ found in such a manner demonstrate reasonable behavior with the changing
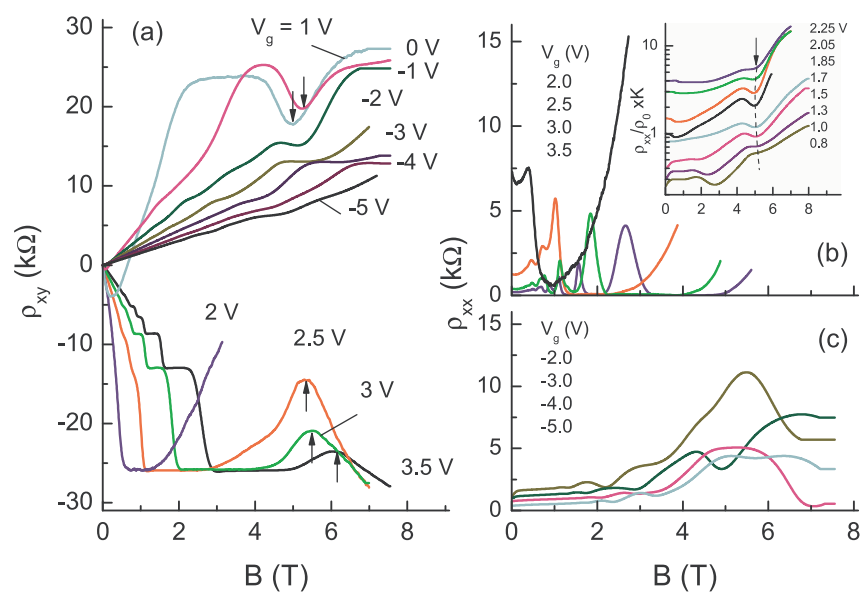

FIG. 2. (Color online) The magnetic field dependencies of $\rho_{x y}$ (a) and $\rho_{x x}(\mathrm{~b}, \mathrm{c})$, measured for the different gate voltages. The minimum resulting from the crossing between the Landau level with $n=0$ of the conduction subband $\mathrm{h} 1$ and Landau level with $n=2$ of the valence subband h2 is marked by an arrow (for more details, see Sec. IV). The inset in (b) illustrates a weak sensitivity of the minimum position to the gate voltage near the charge neutrality point, $V_{g} \simeq 1.8 \mathrm{~V}$. 

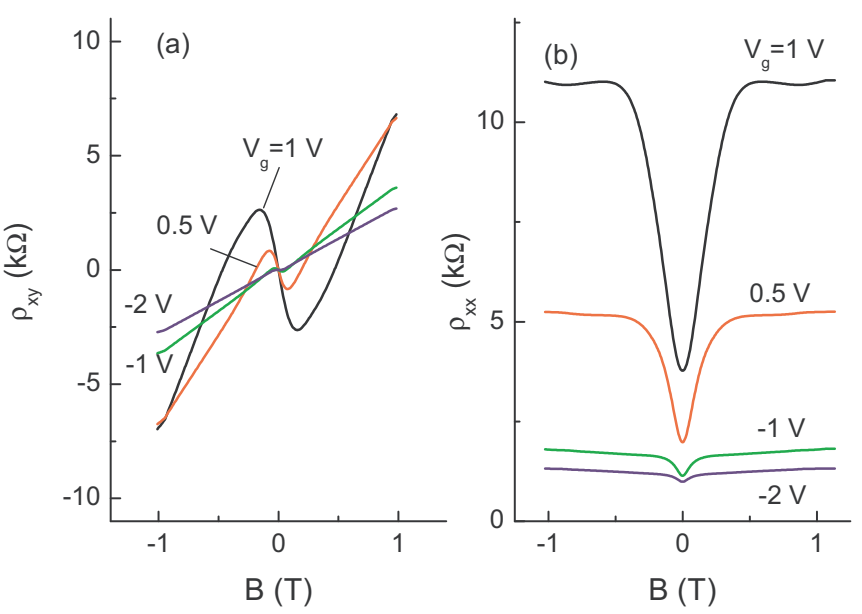

FIG. 3. (Color online) The low-magnetic-field dependencies of $\rho_{x y} \quad$ (a) and $\rho_{x x}$ (b) for different gate voltages illustrating the participation of two types of carriers in the transport.

gate voltage over the whole $V_{g}$ range except for $V_{g}=0-2 \mathrm{~V}$ for electrons and for $V_{g}=1.0-1.5 \mathrm{~V}$ for holes; the hole density linearly decreases, while the electron density linearly increases with the increasing $V_{g}$ value. An important point is that the data points fall on a common straight line with the slope $-5.5 \times 10^{10} \mathrm{~cm}^{-2} \mathrm{~V}^{-1}$, which is close to $-1 / \mathrm{eC}$, where $C=9.1 \mathrm{nF} / \mathrm{cm}^{2}$ is the capacitance between the $2 \mathrm{D}$ gas and gate electrode measured on the same structure. ${ }^{18}$ Thus $-1 / e R_{H}(0.05 \mathrm{~T})$ and $1 / e R_{H}(2 \mathrm{~T})$ give approximate electron and hole densities, respectively, beyond the range of intermediate voltages, $V_{g} \simeq 0-2 \mathrm{~V}$. The carrier densities not

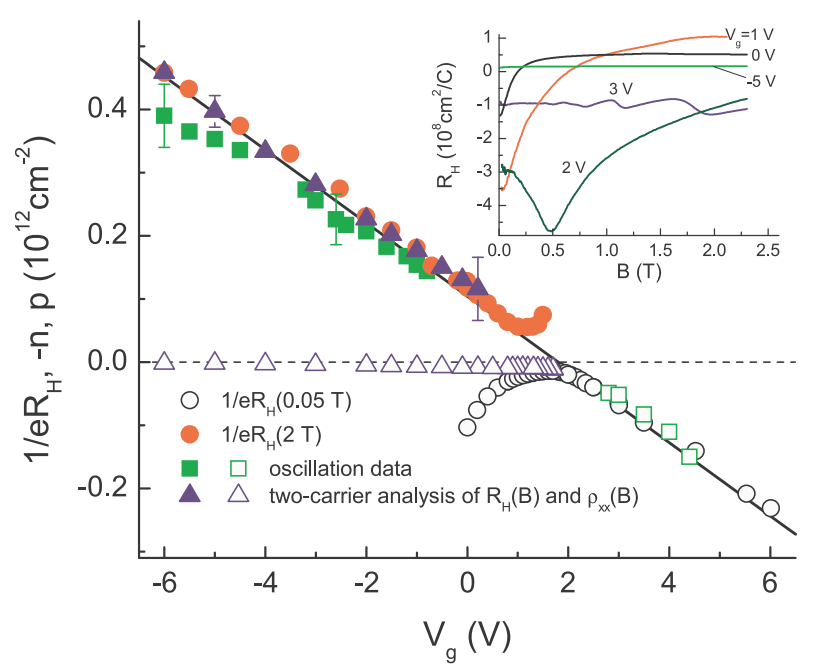

FIG. 4. (Color online) The gate voltage dependence of $1 / e R_{H}(0.05 \mathrm{~T})$ and $1 / e R_{H}(2 \mathrm{~T})$ (circles), of electron and hole densities obtained from analysis of magnetoresistivity oscillations (squares) and from the fit of the $R_{H}$ and $\rho_{x x}$ vs $B$ data within the classical magnetic field range as described in Sec. III C (triangles). Open symbols relate to electrons and filled symbols to holes. For convenience sake, the electron density is negative here. The line is the charge density in the quantum well calculated as $C\left(1.8 \mathrm{~V}-V_{g}\right) / e$, where $C$ is the capacitance between the gate electrode and quantum well measured experimentally, $C=9.1 \mathrm{nF} / \mathrm{cm}^{2}$. The inset shows the $B$ dependencies of the Hall coefficient for several gate voltages. excepting this intermediate range are obtained more precisely by using an analysis of the $B$ dependencies of $R_{H}$ and $\rho_{x x}$ as will be described in Sec. III C. The data presented in Fig. 4 allows one to obtain the $V_{g}$ value, corresponding to charge neutrality point. It is the gate voltage, at which the straight line crosses zero, $V_{g} \simeq 1.8 \mathrm{~V}$.

It is clear that both the additional extremum evident in $\rho_{x x}(B), \rho_{x y}(B)$ at $B=4-6 \mathrm{~T}$ and the alternative sign Hall effect may result from specific features of the energy spectrum of $2 \mathrm{D}$ carries in the structures under study. There are several papers $^{4-6,10,16,19}$ devoted to the study of energy spectrum of the HgTe quantum wells with approximately the same width of the well, $d \simeq 20 \mathrm{~nm}$. However, the energy spectrum, especially of the valence band, is not understood up to now. In what follows, we show that the data obtained from analysis of resistivity oscillations, classical magnetoresistivity and Hall effects demonstrating qualitatively agreement with the spectrum presented in Fig. 1(c) differ from that in quantitative details significantly. We now turn to a close examination of the resistivity oscillations.

\section{B. Resistivity oscillations}

The positions of the oscillation minima in the $\left(B, V_{g}\right)$ plane are plotted in Fig. 5. It is clearly seen that the oscillations resulting from quantization of both electron and hole energy spectra are observed. The oscillations, which minima move to the higher magnetic field with the increasing gate voltage, correspond to electrons. They are evident at $V_{g} \gtrsim 1.5 \mathrm{~V}$. The oscillations of the hole type are observed at $V_{g} \lesssim 1.5 \mathrm{~V}$. They shift to the higher magnetic field when the gate voltage becomes more negative. Figure 5 resembles a fan-chart showing the energies of Landau levels as a function of magnetic field. However, it should be noted that the gate voltage, rather than the energy is plotted in the vertical axis. Only in the

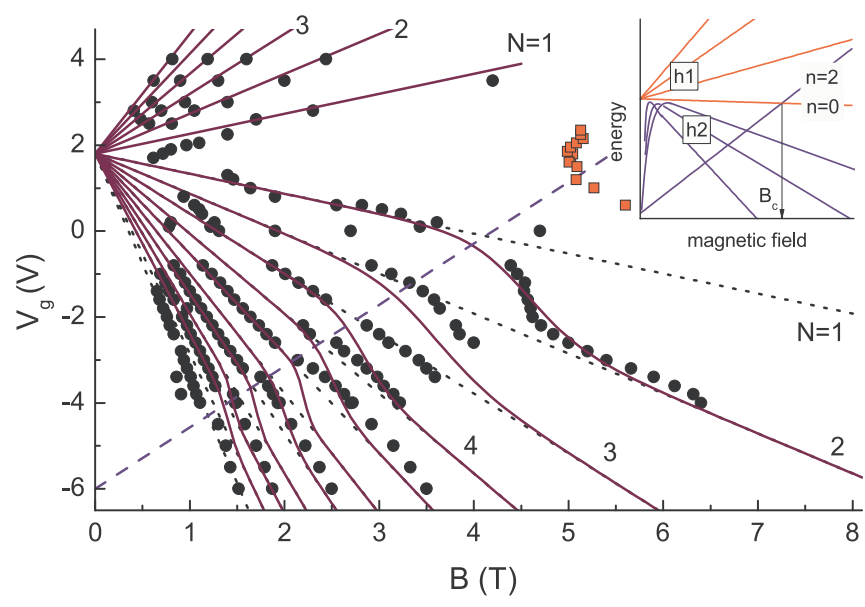

FIG. 5. (Color online) The fan-chart diagram showing the positions of the minima in $\rho_{x x}$ vs $B$ dependence. Symbols are the experimental results. The dashed line is the $B$ dependence of the gate voltage corresponding to a crossing of the Landau level of the h2 subband with $n=2$ with the Fermi level. Solid lines show the expected minima positions. The squares correspond to the minima labeled in Fig. 2(a) by arrows. The inset is schematic dispersion of Landau levels relating to the conduction and valence bands, h1 and $\mathrm{h} 2$, respectively. 
case when the carrier effective mass does not depend on the energy, the Fermi energy varies in direct proportion with $V_{g}$, $E_{F} \propto C V_{g} / e v$, where $v$ is density of states. But even in this case, the vertical scales in the hole and electron parts of the fan-chart will be different due to the different hole and electron effective masses. It is clearly seen that most of the points lying above the dashed line fall on the straight lines, ${ }^{20}$ which are extrapolated at $B \rightarrow 0$ to $V_{g}=(1.8 \pm 0.1) \mathrm{V}$ corresponding to CNP. Just such behavior should be observed when the variation of density of carriers with $V_{g}$ is determined by the geometrical capacitance only. The behavior of $\rho_{x x}$ minima near and below the dashed line will be discussed below.

The hole and electron densities found from the period of the Shubnikov-de Haas $(\mathrm{SdH})$ oscillations in the reciprocal magnetic field for the different gate voltages are depicted in Fig. 4 by squares. As clearly seen, they are close to that found from the Hall effect. It would imply that the Fermi contour in our samples is a single contour centered at $k=0$ both for the electrons and for the holes. For the electrons, this conclusion is natural and commonly accepted. As for the holes, the situation is more delicate and, therefore, we discuss it in more detail.

It is supposed in the number of papers that the valence band is sufficiently warped due to cubic symmetry of the parent materials HgTe and CdTe so that the Fermi contour near the top of the h2 subband comprises four closed contours. Each contour takes the approximate shape of a circle located at $\mathbf{k} \neq 0 .{ }^{6,10,21,22}$ In this case, however, the hole density found from the Hall effect should be four times as large as the density obtained from the $\mathrm{SdH}$ oscillations. Thus, closeness of these values under our conditions means that the warping of the valence band is negligibly small and, hence, the isotropic approximation for the description of the energy spectrum is more adequate for analysis of the data.

As evident from Fig. 1(c), the calculated dispersion of the $\mathrm{h} 2$ subband is nonmonotonic and, therefore, the Fermi contour near the top of the $\mathrm{h} 2$ valence subband takes the shape of two circles centered at $k=0$ within isotropic approximation; the inner circle of radius $k_{F}^{(2)}$ corresponds to the ascending electronlike part of the dispersion $E(k)$ of the $\mathrm{h} 2$ subband, while the outer circle of radius $k_{F}^{(1)}$ corresponds to the descending holelike part. Experimentally, the relation between $k_{F}^{(2)}$ and $k_{F}^{(1)}$ can be obtained from correlation of the Hall effect data with the results obtained from $\mathrm{SdH}$ oscillations. The Hall coefficient for such a dispersion is equal to $1 / e(p-n)$ in the classically high magnetic fields, where $p=\left(k_{F}^{(1)}\right)^{2} / 2 \pi$ and $n=\left(k_{F}^{(2)}\right)^{2} / 2 \pi{ }^{23}$ The period of the hole-type oscillations is determined by the area enclosed by the outer Fermi contour, but not by the inner one. It follows from the fact that the oscillations shift to the higher magnetic field when gate voltages becomes more negative (see Fig. 5). Thus the closeness of the Hall and $\mathrm{SdH}$ hole densities leads us to the conclusion that the radius of the inner Fermi contour is significantly less than that of the outer one, $k_{F}^{(2)} \ll k_{F}^{(1)}$. Some further arguments in support of this conclusion will be presented in Sec. III C.

The measurements of the $\mathrm{SdH}$ oscillations performed for different temperatures give additional information on the energy spectrum. At hole density higher than $10^{11} \mathrm{~cm}^{-2}$ that corresponds to $V_{g}<0 \mathrm{~V}$, one can find the range of low magnetic field, where the spin-unsplit $\mathrm{SdH}$ oscillations

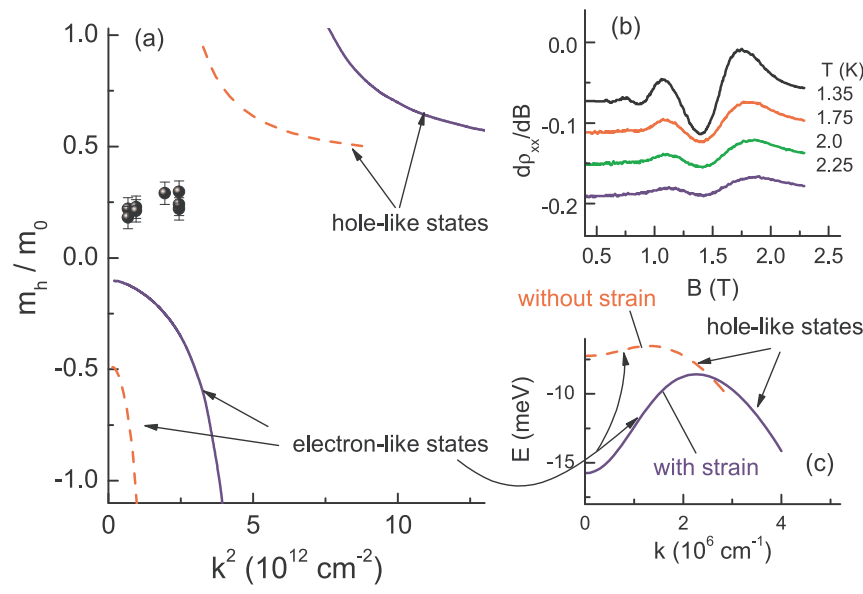

FIG. 6. (Color online) (a) The hole effective mass plotted against the $k^{2}$ value as obtained experimentally (symbols) and calculated theoretically from six-band $k P$ model with and without taking into account the lattice mismatch between $\mathrm{HgTe}$ and CdTe (the sold and dashed curves, respectively). (b) An example of the $\mathrm{SdH}$ oscillations measured for $V_{g}=0.25 \mathrm{~V}\left(p=1.05 \times 10^{11} \mathrm{~cm}^{-2}\right)$ at different temperatures. (c) The dispersion for the hole subband h2 calculated with and without taking into account the strain due to the lattice mismatch (the solid and dashed curves, respectively).

are observed [for example, see Fig. 6(b)]. Therefore fitting the temperature dependence of oscillation amplitude to the Lifshitz-Kosevich formula, ${ }^{24}$ we have found the hole effective mass. We succeeded in such analysis within the density range $p=(1-4) \times 10^{11} \mathrm{~cm}^{-2}$. The results are plotted in Fig. 6(a). One can see that the hole effective mass is equal to $m_{h}=$ $(0.2 \pm 0.05) m_{0}$ at lowest density and only slightly increases with $k_{F} \simeq \sqrt{2 \pi p}$.

Let us compare this result with the result of isotropic six-band $k P$ model. Because the valence band spectrum noticeably depends on the strain, we present in Fig. 6 the dependencies $E(k)$ and $m_{h}(k)=\hbar^{2} k(\partial E / \partial k)^{-1}$ calculated for two cases: with and without taking into account the $\mathrm{HgTe}$ and CdTe lattice mismatch. It is seen that the theory predicts nonmonotonic hole energy spectrum characterized by the electronlike dispersion $E(k)$ with the positive curvature near $k=0$ for both cases. It is significant that the hole effective mass calculated theoretically is negative up to $k^{2} \simeq 2 \times 10^{12}$ or $\simeq 6 \times 10^{12} \mathrm{~cm}^{-2}$ depending on the strain. Experimentally, $m_{h}$ is positive when $k^{2} \gtrsim 0.25 \times 10^{12} \mathrm{~cm}^{-2}$. Note that the values of $m_{h}$ found in our experiments are close to those found from cyclotron resonance. ${ }^{25}$ Thus, the experimental value of the hole effective mass differs drastically from the calculated one to the extent that they are different in sign.

The electron effective mass $m_{e}$ measured by the same way for density range $n=(0.6-1.5) \times 10^{11} \mathrm{~cm}^{-2}$ is equal to $(0.02 \pm 0.005) m_{0}$ that also agrees with the result obtained in the cyclotron resonance experiments. ${ }^{25}$ This value of $m_{e}$ is somewhat smaller than the calculated one, which is equal to $0.028 m_{0}$ and practically independent of the density up to $n=3 \times 10^{11} \mathrm{~cm}^{-2}$.

Thus the parameters of the electron spectrum obtained experimentally from the analysis of the $\mathrm{SdH}$ oscillations are in satisfactory agreement with the calculated ones. As for the energy spectrum of holes, there is discrepancy between the 
data and calculation results. Experimentally, the dispersion of the h2 subband is holelike starting at least from $k \simeq$ $0.5 \times 10^{6} \mathrm{~cm}^{-1}$, while the theory predicts the electronlike dispersion up to sufficiently larger quasimomentum values, $k \simeq 2.5 \times 10^{6} \mathrm{~cm}^{-1}$.

The results obtained from the analysis of magnetoresistivity oscillations do not give an information on the energy spectrum at small $k$ values $k \lesssim 0.5 \times 10^{6} \mathrm{~cm}^{-1}$, and on the overlap of the conduction and valence bands. It can be obtained from the consideration of the classical low-field magnetoresistivity and Hall effect.

\section{Classical transport}

The detailed measurements at low magnetic field show that the Hall resistivity $\rho_{x y}$ is strongly nonlinear at $B<0.1-$ $0.5 \mathrm{~T}$ insofar that it changes the sign [see Fig. 3(a)]. The large positive magnetoresistivity is observed within this magnetic field range [see Fig. 3(b)]. Both these facts strongly suggest that at least two types of carriers, electrons and holes, take part in the transport.

The are several physical reasons that can manifest themselves as a second type of carriers in transport. They are the following: existence of edge states ${ }^{2,8,26-28}$ or inversion "layers" like those existing on the surface of the bulk $\mathrm{Hg}_{1-x} \mathrm{Cg}_{x} \mathrm{Te}$ samples, ${ }^{29}$ which can give the electron contribution to the conductivity, while the 2D gas is of hole type; existence of 2D electron and hole drops due to fluctuations of electrostatic potential, quantum well width, composition of the barriers, etc; existence of conductivity channels of technological nature located outside the quantum well, e.g., near the $\mathrm{Hg}_{0.42} \mathrm{Cd}_{0.58} \mathrm{Te} / \mathrm{CdTe}$ interfaces; finally, the overlapping between the conduction and valence bands predicted by the standard $k P$ model. In Ref. 30, we consider this issue in great detail. We show that the first three mechanisms are ineffective in our case and several types of carriers are due to the overlap between the subbands.

It is natural in this case to use the classical handbook formulas for the conductivity by two types of carriers (see, e.g., Ref. 31) to fit the magnetic field dependencies of $\rho_{x x}$ and $R_{H}$. Such fitting procedure has been performed at a low magnetic field, $B<0.3 \mathrm{~T}$, with the use of densities and mobilities of electrons and holes as fitting parameters. As an example we show the results of the best fit for $V_{g}=1.1$ and $-2 \mathrm{~V}$ in Fig. 7. It is seen that this simple model quite well describes both dependencies, $\rho_{x x}(B)$ and $R_{H}(B)$. Some inconsistency between experimental and fitting curves evident in the high magnetic field domain may result from the change of the carrier densities or/and mobilities with a growing magnetic field, which is ignored under our analysis.

First, we consider the gate voltage dependencies of the hole and electron densities found by this manner. They are shown in Fig. 4 by triangles. One can see that the hole density agrees well with that obtained from the analysis of the resistivity oscillations and practically coincides with the $1 / e R_{H}(2 \mathrm{~T})$ value. As for the electron density, it is much less than the hole one. In the larger scale, the $n$ versus $V_{g}$ dependence is plotted in Fig. 8(a). As seen in this figure, it is rather complicated. Three $V_{g}$ intervals corresponding to the different positions of the Fermi level can be distinguished: (1)
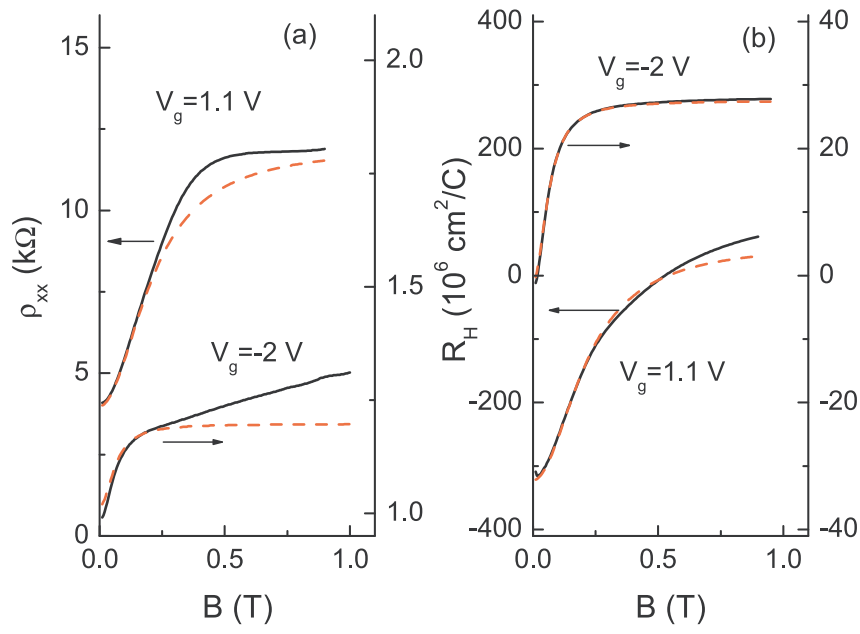

FIG. 7. (Color online) The magnetic field dependencies of $\rho_{x x}$ (a) and $R_{H}$ (b) in the regime when both the holes and the electrons take part in the transport. The solid curves are measured experimentally, the dashed lines are the results of the best fit to the classical formula.

at $V_{g}>1.8 \mathrm{~V}$, the electron density decreases with decreasing $V_{g}$ with the rate $K^{(1)}=\Delta n / \Delta V_{g}$ of about $5.5 \times 10^{10} \mathrm{~cm}^{-2}$ $\mathrm{V}^{-1}$. The Fermi level over this $V_{g}$ range lies in the conduction h1 subband, and the Hall effect and the conductivity are solely determined by the electrons of this subband. (2) At $V_{g}=1.0-1.7 \mathrm{~V}, \Delta n / \Delta V_{g}=K^{(2)}$ is about ten times less than that at $V_{g}>1.8 \mathrm{~V}, K^{(2)} \simeq 6.0 \times 10^{9} \mathrm{~cm}^{-2} \mathrm{~V}^{-1}$ [see inset in Fig. 8(a)]. Quite apparently this feature results from the fact that the Fermi level is lowered into the valence band, and electrons and holes coexist within this region. In this case, the rate of decrease of the Fermi energy is mainly determined by the hole density of states, which is larger than the electron one. If so, the relation between the slopes within the intervals (1) and (2) should be determined by the relation between the hole and electron densities of
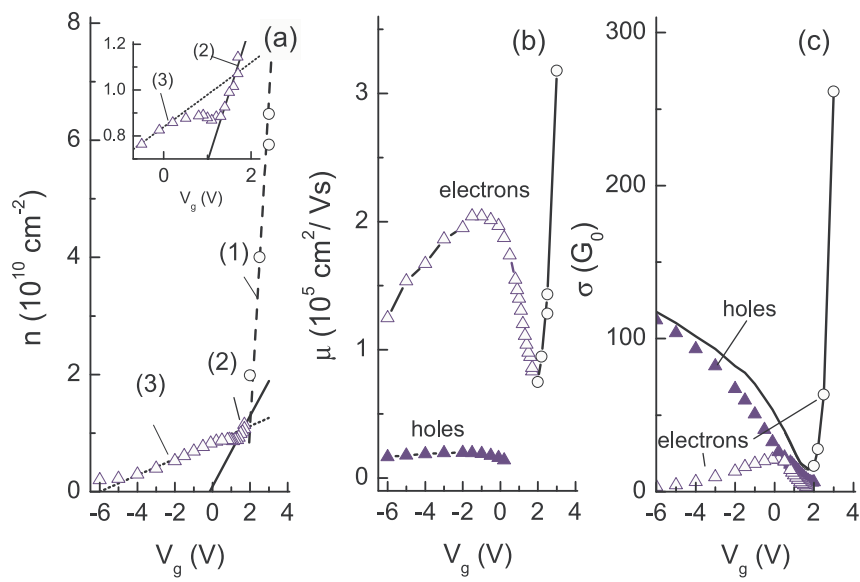

FIG. 8. (Color online) The gate voltage dependencies of (a) electron density, (b) electron mobility, and (c) partial electron and hole conductivities. The parameters of carriers shown by triangles are obtained within the framework of standard two type carrier model as described in the text, the parameters shown by circles are obtained as follows: $n=1 / e\left|R_{H}(0.05 \mathrm{~T})\right|, \mu_{e}=\left|R_{H}(0.05 \mathrm{~T})\right| \sigma$. The solid line in (c) is the $\sigma$ vs $V_{g}$ dependence measured experimentally. 
states: $\quad K^{(1)} / K^{(2)}=d E_{F} /\left.d V_{g}\right|_{V_{g}>1.8 \mathrm{v}} / d E_{F} /\left.d V_{g}\right|_{V_{g}<1.8 \mathrm{~V}}=$ $1+v_{h} / v_{e} \simeq 1+m_{h} / m_{e}$, where approximate equality is written under assumption that the hole density of states is not markedly affected by the electronlike states. If one uses the experimental values of hole and electron effective masses $m_{h} \simeq 0.2 m_{0}$ and $m_{e} \simeq 0.02 m_{0}$ (see Sec. III B), we obtain $K^{(1)} / K^{(2)} \simeq 11$ that practically coincides with the experimental value. Thus it is safe to assume that the electron contribution to the transport comes mainly from the conduction band electrons in this region. If one extrapolates the $n$ versus $V_{g}$ plot to $n=0$ as shown in Fig. 8(a) by the solid line, one can obtain the gate voltage where the electrons of the conduction band are expected to disappear. As seen it should occur at $V_{g} \approx 0 \mathrm{~V}$. (3) Within the $V_{g}$ range from $0 \mathrm{~V}$ down to $-6 \mathrm{~V}$, the electron contribution to the conductivity remains essential. The data points in this range fall on the straight line with the slope $K^{(3)} \simeq 1.5 \times 10^{9} \mathrm{~cm}^{-2} \mathrm{~V}^{-1}$. Because the conduction band electrons have to disappear at $V_{g} \approx 0 \mathrm{~V}$, it remains to assume that the electron contribution at these gate voltages comes from the holes with electronlike energy spectrum in the vicinity of $k=0$.

We switch now attention to the gate voltage dependence of the electron mobility [see Fig. 8(b)]. As clearly seen in Fig. 8(b), it is nonmonotonic; a sharp minimum is evident near $V_{g} \simeq 1.8 \mathrm{~V}$. At $V_{g}>1.8 \mathrm{~V}$, when the conductivity is determined by the conduction band electrons, while the holes in the valence band are absent, the mobility decreases with the decreasing gate voltage. This is natural because the decrease of the electron density and, hence, of the electron energy leads to the increase of scattering probability independently of whether a short- or a long-range scattering potential determines the mobility. The increase of electron mobility with decreasing $V_{g}$ at $V_{g}<1.8 \mathrm{~V}$, where the electron density carries on decreasing, seems strange at first sight. However, it can be explained by holes appearing at these gate voltages, which screen the potential of scatterers effectively due to the large effective mass. The authors of Ref. 32 who observed analogous behavior of the electron mobility come to the same conclusion.

Let us summarize the results of this section. First, the electron and hole subbands, h1 and h2, respectively, are overlapped. Second, the dispersion $E(k)$ of the $\mathrm{h} 2$ subband is really nonmonotonic, being holelike at large quasimomentum values it demonstrates electronlike behavior near $k=0$. Schematically, such a spectrum is displayed in Fig. 9. The transport properties are strongly dependent on the position of the Fermi level. At high gate voltage, $V_{g} \gtrsim 1.8 \mathrm{~V}$, the Fermi level lies within the $\mathrm{h} 1$ conduction subband (region A in Fig. 9). The transport is fully determined by electrons occupying this subband. As the gate voltage decreases, the Fermi level moving down crosses the top of the valence $\mathrm{h} 2$ subband at $V_{g} \simeq 1.8 \mathrm{~V}$ and enters the area $\mathrm{B}$ where the conduction and valence band are overlapped. The transport in this regime should be determined by three types of carriers. They are electrons of the h1 subband, and two types of positively charged carriers of $\mathrm{h} 2$ subband characterized by the hole- and electronlike dispersion. The carriers of the first and third types give the negative contribution to the Hall effect, while the contribution of the second type carriers is positive. We believe that the contribution of the holes with electronlike dispersion

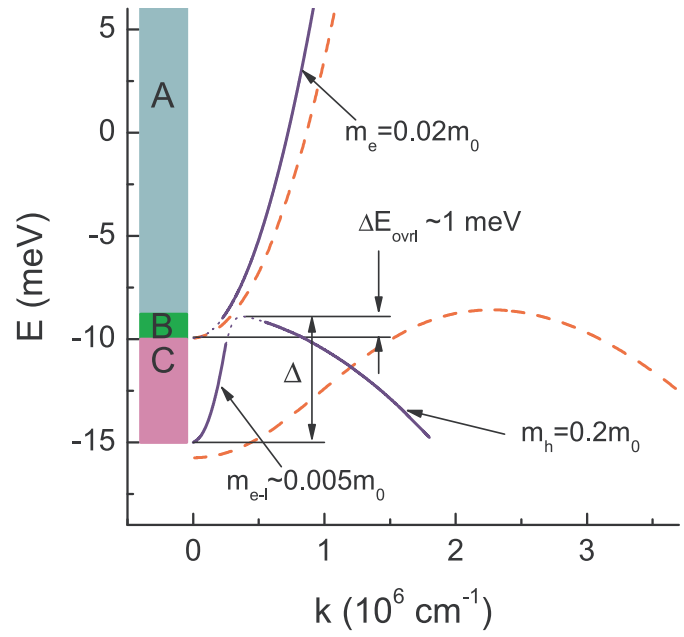

FIG. 9. (Color online) The dispersion $E(k)$ reconstructed from the data analysis as described in the text (solid lines) and calculated within the framework of isotropic six-band $k P$ model (dashed lines). The dotted parts of dispersion curves show an assumed run of the dispersion curves not supported experimentally.

to the magnetotransport is small at $V_{g}=(1.8-1.0) \mathrm{V}$ in our concrete case and becomes significant at $V_{g} \lesssim 1 \mathrm{~V}$. Finally at $V_{g}<0 \mathrm{~V}$, when the Fermi level is below the h1 subband bottom (region C in Fig. 9), only the carriers of the h2 subband take part in the transport.

\section{THE SPECTRUM: COMPARISON BETWEEN THEORY AND EXPERIMENT}

Let us reconstruct the energy spectrum of the electron and hole subbands using the experimental results presented in Secs. III B and IIIC (see Fig. 9). As shown in the previous section, the conduction and valence bands are overlapped. The value of overlapping ( $\left.\Delta E_{\text {ovrl }}\right)$ can be estimated from the value of the electron density at the gate voltage corresponding to the appearance of the hole contribution to the conductivity. Inspection of Fig. 8(c) shows that this happens at $V_{g} \simeq 1.8 \mathrm{~V}$. As seen from Fig. 8(c), the electron density $n$ at this gate voltage is about $1 \times 10^{10} \mathrm{~cm}^{-2}$, which gives $\Delta E_{\mathrm{ovrl}} \simeq n / v_{e} \sim$ $1 \mathrm{meV}$ if one uses the experimental value of electron effective mass $m_{e}=0.02 m_{0}$ to calculate the electron density of states $v_{e}$. This overlap value is five time as small as that obtained experimentally in Refs. 21 and 22. It should be noted that the observable overlap consists of two contributions. The first contribution is the band overlap due to specifics of the energy spectrum of the ideal heterostructure without disorder. The second one is the overlap caused by disorder. It can be disorder of different origin, e.g., caused by fluctuations of the electrostatic potential, the quantum well width, or cadmium content in the barriers. We believe that the smallest experimental value $\Delta E_{\mathrm{ovrl}} \simeq 1 \mathrm{meV}$ corresponds to the subband overlap in the ideal heterostructure more adequately.

Experimentally, the dispersion $E(k)$ of the valence $\mathrm{h} 2$ subband is monotonic and holelike at $p \gtrsim 5 \times 10^{10} \mathrm{~cm}^{-2}$, which corresponds to $k=(2 \pi p)^{1 / 2} \gtrsim 0.5 \times 10^{6} \mathrm{~cm}^{-1}$ (see Sec. III B). The effective mass obtained from the analysis of 
$\mathrm{SdH}$ oscillations is independent of $k$ and close to $0.2 m_{0}$. For lower $k$ values, $k \lesssim 0.5 \times 10^{5} \mathrm{~cm}^{-1}$, the energy spectrum is electronlike, i.e., there is a narrow minimum in the dispersion $E(k)$ (see Sec. IIIC). The energy of this minimum with reference to the top of valence band $(\Delta)$ can be easily estimated from the hole density at the gate voltage $V_{g} \simeq-6 \mathrm{~V}$, which corresponds to disappearance of the electron contribution [see Fig. 8(a)]: $\Delta=p(-6 \mathrm{~V}) / v_{h}$. With the use of the experimental value of the hole effective mass, $m_{h}=0.2 m_{0}$, and $p(-6 \mathrm{~V}) \simeq$ (4-5) $\times 10^{11} \mathrm{~cm}^{-2}$ (see Fig. 4), one obtains $\Delta \simeq 5 \mathrm{meV}$. The effective mass of electronlike states of the valence band is about $m_{e-l} \simeq-0.005 m_{0}$. This estimate is obtained from the $\Delta$ and $\Delta E_{\text {ovrl }}$ values and from the amount of electronlike states at the gate voltage $V_{g} \simeq 0 \mathrm{~V}$, at which the Fermi level escapes the conduction band, $n(0 \mathrm{~V}) \simeq 8 \times 10^{9} \mathrm{~cm}^{-2}$ [see the inset in Fig. 8(a)]: $\left|m_{e-l}\right| \simeq \pi \hbar^{2} n(0 \mathrm{~V}) /\left(\Delta-\Delta E_{\text {ovrl }}\right)$.

The energy spectrum reconstructed with the use of the parameters $m_{e}, m_{h}, m_{e-l}, \Delta$, and $\Delta E_{\text {ovrl }}$ estimated above is shown by the solid lines in Fig. 9. The parts of dispersion $E(k)$ not supported experimentally are drawn by the dotted lines. In the same figure, the spectrum calculated in the framework of the $k P$ model is depicted. One can see that the $E$ versus $k$ dependence of the conduction band is close to the theoretical one. The difference between the dispersion curves $E(k)$ for the valence band is crucial.

Additional arguments in favor of the energy spectrum shown in Fig. 9 are obtained from analysis of the data in high magnetic field. As noted in Sec. III A, an additional extremum marked by the arrows in Figs. 2(a) and 2(b) is observed on the experimental dependencies $\rho_{x y}(B)$ and $\rho_{x x}(B)$ at the gate voltages near CNP. Its position in the $\left(B, V_{g}\right)$ coordinates is shown in Fig. 5 by squares. The origin of the extremum relates directly to specific features of the magnetic field quantization of the energy spectrum in the HgTe quantum well with inverted spectrum. ${ }^{33}$ There are two singular Landau levels responsible for that [see Fig. 1 in Ref. 33 and sketch in the inset in Fig. 5]. They are the lowest level of the conduction band with $n=0$ and the level of valence h2 subband with the number $n=2$. Unusual behavior of these two levels leads to a crossing of the conduction- and valence-band states at some value of the magnetic field, $B=B_{c}$. Thus, for the case when $n$ is slightly larger than $p$, the Fermi level lies in the Landau level $n=0$ of the h1 subband at $B \lesssim B_{c}$, while at the higher magnetic field, $B \gtrsim B_{c}$, the Fermi level occurs in the Landau level $n=2$ of the h2 subband resulting, thus, in switching of the electron ground state and in the peculiar behavior of $\rho_{x x}$ and $\rho_{x y}$ with the growing magnetic field. Analogous switching happens when $p$ is slightly larger than $n$. Such an effect in HgTe quantum wells of different widths has been earlier investigated in Refs. 8,33, and 34. If one extrapolates the calculation results 8,33 to $d=20$ nm, we obtain $B_{c} \simeq 4-5 \mathrm{~T}$, which is close to the position of the peculiarities evident in the $\rho_{x x}$ and $\rho_{x y}$ versus $B$ dependencies in our experiments.

Unusual behavior of the Landau level $n=2$ reveals itself in unusual behavior of the oscillation maxima with the increasing hole density. As seen from Fig. 5, the data points lying above the dashed line in the hole part of the fan chart fall on the straight lines drawn in accordance with the usual condition $p=e B / h \times N$, where $h$ is the Plank constant, $N=1,2, \ldots$, and $p=-5.5 \times 10^{10}\left(V_{g}-1.8 \mathrm{~V}\right) \mathrm{cm}^{-2}$ (see Fig. 4). The points lying below the dashed line deviate or tend to deviate from these lines. This is more pronounced for the minimum whose position follows the line with $N=1$ at $B \lesssim 4 \mathrm{~T}$, and it jumps to the line with $N=2$ at $B \simeq 4.5 \mathrm{~T}$. Only the singular Landau level $n=2$ is responsible for such an anomaly because it moves in the opposite direction as compared with the other hole Landau levels in the high magnetic field (see inset in Fig. 5). If one traces the concrete $\rho_{x x}$ minimum, corresponding to the location of the Fermi level between the given normal Landau levels, we obviously obtain that its position being determined by the filling factor $N$ in the low magnetic field will be determined by the filling factor $N+1$ in the higher magnetic field when the singular Landau level becomes above the Fermi level. Therefore the oscillation minima should in fact follow the solid curves as shown schematically in the lower part of the fan chart in Fig. 5. Thus this model describes the behavior of the oscillation minima rather well. Some discrepancy is not surprising because this simple consideration is valid when the overlapping between the Landau levels is small, while the experimental data were obtained within a wide magnetic field range involving both the $\mathrm{SdH}$ and quantum Hall regimes.

Because the energy of the Landau level $n=2$ tends to the energy of the h2 subband at $k=0$ when $B$ tends to zero, we are able to estimate the position of the valence $\mathrm{h} 2$ subband at $k=0$ independently. As follows from the above analysis, the dashed line in Fig. 5 is the $V_{g}$ dependence of the magnetic field, in which the Landau level $n=2$ crosses the Fermi level. With $B$ tending to zero, this line is extrapolated to $V_{g} \simeq-(5-6) \mathrm{V}$. It means that the Fermi level passes through the minimum of the h2 subband when $V_{g} \simeq-(5-6) \mathrm{V}$. Then, the energy of the h2 subband at $k=0$ measured from the top of the $\mathrm{h} 2$ subband is approximately equal to the Fermi energy found from the hole density at this gate voltage: $\Delta=p / v_{h} \simeq 4.5-5.5 \mathrm{meV}$. This value is consistent with that estimated above from the hole density at the gate voltage corresponding to the disappearance of the electron contribution to the conductivity.

\section{CONCLUSION}

We have studied the transport phenomena in a HgTe single quantum well with the inverted energy spectrum. Consistent analysis of the magnetic field dependencies of the magnetoresistivity, the Hall coefficient, and the SdH effect in gated samples carried out over a wide range of the electron and hole densities including the vicinity of charge neutrality point allows us to reconstruct the structures of the electron and hole subbands of spatial quantization, h1 and h2, respectively. It has been shown that the reconstructed energy spectrum is in qualitative agreement with the spectrum calculated in the framework of standard $k P$ approach. The $\mathrm{h} 1$ and $\mathrm{h} 2$ subbands are overlapped resulting in a two-dimensional semimetal. The calculated and experimental effective masses of the electron h1 subband are close to each other.

The key result of the paper, however, is that the quantitative difference between the experimental and calculated dispersion of the hole h2 subband is drastic. We have experimentally shown that the curvature of the $\mathrm{h} 2$ subband is holelike starting from $k \simeq 0.5 \times 10^{6} \mathrm{~cm}^{-1}$. This result is in conflict with the theory, which predicts the electronlike dispersion with 
the negative effective mass up to significantly larger quasimomentum value, $k \simeq 2.5 \times 10^{6} \mathrm{~cm}^{-1}$. The hole effective mass measured experimentally at $k \simeq(0.8-1.6) \times 10^{6} \mathrm{~cm}^{-1}$ is equal to approximately $0.2 m_{0}$, which is far less than that calculated theoretically for any quasimomentum value. The experimentally obtained effective mass near $k=0$, where the spectrum is electronlike, is close to $-0.005 m_{0}$, whereas the theory predicts the absolute value larger than $0.1 m_{0}$.

Noteworthy is that we have ignored the interaction between electrons and holes when interpreting the data and reconstructing the energy spectrum. The applicability of the singleparticle approximation for the description of the low density electron gas $\left(n \sim 10^{9} \mathrm{~cm}^{-2}\right.$ ) existing on the background of a hole gas of much higher density $\left(p \sim 10^{11} \mathrm{~cm}^{-2}\right)$ is not so apparent. All this indicates that further experimental and theoretical investigations are needed to find the answer to the question of whether the standard $k P$ model adequately describes the energy spectrum of wide $\mathrm{HgTe}$-based single quantum wells.

\section{ACKNOWLEDGMENTS}

This work has been supported in part by the RFBR (Grant Nos. 11-02-12126, 12-02-00098, and 13-02-00322).
${ }^{1}$ L. G. Gerchikov and A. Subashiev, Phys. Status Solidi B 160, 443 (1990).

${ }^{2}$ B. A. Bernevig, T. L. Hughes, and S.-C. Zhang, Science 314, 1757 (2006).

${ }^{3}$ M. I. D'yakonov and A. Khaetskii, Zh. Eksp. Teor. Fiz. 82, 1584 (1982) [Sov. Phys. JETP 55, 917 (1982)].

${ }^{4}$ G. Landwehr, J. Gerschütz, S. Oehling, A. Pfeuffer-Jeschke, V. Latussek, and C. R. Becker, Physica E 6, 713 (2000).

${ }^{5}$ X. C. Zhang, A. Pfeuffer-Jeschke, K. Ortner, C. R. Becker, and G. Landwehr, Phys. Rev. B 65, 045324 (2002).

${ }^{6}$ K. Ortner, X. C. Zhang, A. Pfeuffer-Jeschke, C. R. Becker, G. Landwehr, and L. W. Molenkamp, Phys. Rev. B 66, 075322 (2002).

${ }^{7}$ X. C. Zhang, K. Ortner, A. Pfeuffer-Jeschke, C. R. Becker, and G. Landwehr, Phys. Rev. B 69, 115340 (2004).

${ }^{8}$ M. König, S. Wiedmann, C. Brüne, A. Roth, H. Buhmann, L. W. Molenkamp, X.-L. Qi, and S.-C. Zhang, Science 318, 766 (2007).

${ }^{9}$ G. M. Gusev, Z. D. Kvon, O. A. Shegai, N. N. Mikhailov, S. A. Dvoretsky, and J. C. Portal, Phys. Rev. B 84, 121302 (2011).

${ }^{10}$ Z. D. Kvon, E. B. Olshanetsky, E. G. Novik, D. A. Kozlov, N. N. Mikhailov, I. O. Parm, and S. A. Dvoretsky, Phys. Rev. B 83, 193304 (2011).

${ }^{11}$ G. Tkachov and E. M. Hankiewicz, Phys. Rev. B 84, 035444 (2011).

${ }^{12}$ J. W. Nicklas and J. W. Wilkins, Phys. Rev. B 84, 121308 (2011).

${ }^{13}$ P. M. Ostrovsky, I. V. Gornyi, and A. D. Mirlin, Phys. Rev. B 86, 125323 (2012).

${ }^{14}$ N. N. Mikhailov, R. N. Smirnov, S. A. Dvoretsky, Y. G. Sidorov, V. A. Shvets, E. V. Spesivtsev, and S. V. Rykhlitski, Int. J. Nanotechnology 3, 120 (2006).

${ }^{15}$ V. A. Larionova and A. V. Germanenko, Phys. Rev. B 55, 13062 (1997).

${ }^{16}$ X. C. Zhang, A. Pfeuffer-Jeschke, K. Ortner, V. Hock, H. Buhmann, C. R. Becker, and G. Landwehr, Phys. Rev. B 63, 245305 (2001).

${ }^{17}$ E. G. Novik, A. Pfeuffer-Jeschke, T. Jungwirth, V. Latussek, C. R. Becker, G. Landwehr, H. Buhmann, and L. W. Molenkamp, Phys. Rev. B 72, 035321 (2005).

${ }^{18}$ The capacitance measured is practically independent of the gate voltage over the entire $V_{g}$ range. Only one percent variation of $C$ resulting from the finite value of compressibility of the electron gas is evident at $V_{g} \approx 1.8 \mathrm{~V}$.

${ }^{19}$ M. S. Zholudev, A. V. Ikonnikov, F. Teppe, M. Orlita, K. V. Maremyanin, K. E. Spirin, V. I. Gavrilenko, W. Knap, S. A. Dvoretskiy, and N. N. Mihailov, Nanoscale Res. Lett. 7, 534 (2012).
${ }^{20}$ The deviation of the points with $N=1$ from the corresponding straight line evident in the electron part of the fan chart at $V_{g}=1.7-2.0 \mathrm{~V}$ is due to the fact that the lines are drawn under the assumption that only the electrons with a density proportional to the gate voltage are present in the quantum well. This assumption becomes invalid in the vicinity of charge neutrality point where both electrons and holes with close densities take part in the transport.

${ }^{21}$ Z. D. Kvon, E. Olshanetsky, D. A. Kozlov, N. N. Mikhailov, and S. A. Dvoretskii, Pis'ma Zh. Eksp. Teor. Fiz. 87, 588 (2008) [JETP Lett. 87, 502 (2008)].

${ }^{22}$ E. B. Olshanetsky, Z. D. Kvon, M. V. Entin, L. I. Magarill, N. N. Mikhailov, I. O. Parm, and S. A. Dvoretskii, Pis'ma Zh. Eksp. Teor. Fiz. 89, 338 (2009) [JETP Lett. 89, 290 (2009)].

${ }^{23}$ B. M. Askerov, Electron Transport Phenomena in Semiconductors (World Scientific, Singapore, 1994), p. 394.

${ }^{24}$ I. M. Lifshits and A. M. Kosevich, Zh. Eksp. Teor. Fiz. 29, 730 (1955) [Sov. Phys. JETP 2, 636 (1956)].

${ }^{25}$ Z. D. Kvon, S. N. Danilov, D. A. Kozlov, C. Zoth, N. N. Mikhailov, S. A. Dvoretskii, and S. D. Ganichev, Pis'ma Zh. Eksp. Teor. Fiz. 94, 895 (2011) [JETP Lett. 94, 816 (2011)].

${ }^{26}$ A. Roth, C. Brüne, H. Buhmann, L. W. Molenkamp, J. Maciejko, X.-L. Qi, and S.-C. Zhang, Science 325, 294 (2009).

${ }^{27}$ T. L. Schmidt, S. Rachel, F. von Oppen, and L. I. Glazman, Phys. Rev. Lett. 108, 156402 (2012).

${ }^{28}$ J. I. Väyrynen, M. Goldstein, and L. I. Glazman, Phys. Rev. Lett. 110, 216402 (2013).

${ }^{29}$ V. F. Radantsev, T. I. Deryabina, G. I. Kulaev, and E. L. Rumyantsev, Phys. Rev. B 53, 15756 (1996).

${ }^{30}$ See Supplemental Material at http://link.aps.org/supplemental/ 10.1103/PhysRevB.88.155306 for analysis of physical reasons that can manifest themselves as a second type of carriers.

${ }^{31}$ F. J. Blatt, Physics of Electronic Conduction in Solids (McGrawHill, US, 1968), p. 446.

${ }^{32}$ E. Olshanetsky, Z. Kvon, N. Mikhailov, E. Novik, I. Parma, and S. Dvoretsky, Solid State Commun. 152, 265 (2012).

${ }^{33}$ M. Schultz, U. Merkt, A. Sonntag, U. Rössler, R. Winkler, T. Colin, P. Helgesen, T. Skauli, and S. Løvold, Phys. Rev. B 57, 14772 (1998).

${ }^{34}$ O. E. Raichev, G. M. Gusev, E. B. Olshanetsky, Z. D. Kvon, N. N. Mikhailov, S. A. Dvoretsky, and J. C. Portal, Phys. Rev. B 86, 155320 (2012). 